\section{Dispersal via whale bones}

SIR - Smith et al. ${ }^{1}$ reported the occurrence of chemosynthetic invertebrates, especially molluscs, associated with whale bones on the Pacific Ocean floor. More recently, Squires et $a l^{2}$ have given an account of fossil whale carcasses from Oligocene deep-water sediments also associated with chemosynthetic molluscs, and discussed the possibility of whale carcasses acting as stepping stones for the wide dispersal of chemosynthetic invertebrates over a prolonged period. But Squires et al. argue that carcasses large enough to act as benthic stepping stones have existed in the Pacific Basin only since the late Miocene, that is, for the past 11 million years. We believe the idea that only whale carcasses can act as refugia is unduly restrictive. The oceans have contained large marine tetrapods since at least the end of the Triassic, in other words for around 200 million years. By large, we mean animals with body lengths (conservatively estimated) of up to $10 \mathrm{~m}$. In addition, large fishes also up to $10 \mathrm{~m}$ long are known from at least as early as the Middle and Upper Jurassic to the present day.

Associations of fossil marine-reptile skeletons with abundant invertebrates have been demonstrated. For example, a specimen of the ichthyosaur Ophthalmosaurus was associated with specimens of the small scaphopod Prodentalium ${ }^{3}$, a taxon reported associated with Eocene methane vents ${ }^{4}$; and a complete plesiosaur skeleton was associated with articulated valves of Nicaniella (Trautscholdia) sp. from the Oxford Clay (Callovian, Middle Jurassic), an organic rich mudrock of shallow water facies. This deposit is itself rich in early diagenetic pyrite, indicating considerable sulphate reduction in the top few millimetres of the sea floor.

If vertebrate carcasses are important in the dispersal of vent faunas, then such refugia may have occurred from at least the end of the Middle Triassic, when large ichthyopterygians first appeared, or from the end of the Triassic, when large sauropterygians made their first appearance. The ichthyosaurs disappeared during the Middle Cretaceous, but sauropterygians and mosasaurs remained important parts of the marine fauna until the end of the Mesozoic. Gigantic fishes have been present in the seas since the Devonian. Large fishes, including teleosts and elasmobranchs, spanned the Cretaceous/Tertiary boundary ${ }^{5}$, and may have provided refugia before the arrival of cetaceans in the Eocene.

Nevertheless, caution should be exercised when examining fossils associated with vertebrates preserved in concretions. Early diagenesis around carcasses may preferentially preserve calcareous invertebrates that may be absent from the surrounding sediment due to diagenetic removal. In addition, preparators of vertebrate fossils may inadvertently destroy invertebrates. We suggest that palaeontologists look more closely at the skeletons of large marine vertebrates of all sorts, and especially their associated invertebrates.

D. M. MARTILL

A. R. I. Cruickshank

Department of Earth Sciences,

The Open University,

Milton Keynes MK7 6AA, UK

Earth Sciences Section,

M. A. TAYLOR

Leicestershire Musems, Arts and

Records Service,

Leicester LE1 6TD, UK

1. Smith, C. R. et al. Nature 341, 27-28 (1989).

2. Squires, R. L., Goedert, J. L. \& Barnes, L. G. Nature 349, 574 (1991).

3. Martill, D. M. Palaeontology 30, 543-556 (1987).

4. Goedert, J. L. \& Squires, R. L. Geology 18, 1182-1185 (1990)

5. Harland, W. B. et. al. The Fossil Record: A Symposium With Documentation (Geological Society, London, 1967).

\section{Human circadian rhythms \\ SIR - Winfree in his News and Views ${ }^{1}$ on our} paper $^{2}$ outlined the development of the theory that underlies the concept of the phase-free singular condition of a physiological rhythm generator, but in doing so he understated his own contribution. He has articulated lucidly the mathematical necessity of the singular state ${ }^{3}$, developed an experimental protocol (the singularity trap) by which the timing and strength of the critical stimulus can be bounded, and applied this protocol to a circadian rhythm of Drosophila melanogaster ${ }^{4}$. But Winfree's use of the terms "subjective midnight" and "subjective noon" to calibrate the response of the human circadian clock to light could be misunderstood.

We have shown ${ }^{2}$ that, for persons reasonably well entrained to the conventional day the critical light stimulus is centred at 4:00 to 5:00 a.m. This time also represents the transition between those light-induced phase shifts that retard the circadian clock (light episodes before 4:00 a.m.) and those that advance it (episodes after 5:00 a.m.). The complementary time for transition from advances to retardations is 4:00-5:00 p.m. Thus, Winfree's subjective midnight is, for humans, about 4:30 a.m., whereas subjective noon is about 4:30 p.m. That inconsistency may in part be because Winfree has chosen to use the onset of the stimulus as its reference time. For the relatively brief light stimuli which are effective in many insects and nocturnal rodents, the distinction between onset time and mid-stimulus time is small. But for the 5- to 9-hour range of stimuli that we used, consistency is obtained only if the centre of the stimulus episode is taken as its reference. This was also the practice of Gander and Lewis ${ }^{5}$ when using 4- to 16-hour light episodes as stimuli for Rattus exulans.
We urge that the 'subjective noon' and 'subjective midnight' nomenclature be avoided with reference to humans because of its susceptibility to misinterpretation by the untutored reader. Indeed, it can be misleading even to experts. Before any data were available on human resetting by light, Daan and Lewy ${ }^{6}$ conjectured that the human response might qualitatively resemble the well-documented type 1 response of nocturnal rodents. They consequently assumed that subjective midnight for humans was midnight, thereby misestimating the true critical time for humans by 4 to 5 hours.

Like Winfree, we are struck by the uniformity of response characteristics across species, but the precise relation of critical time to clock time depends both on the species and its environmental light exposure. In humans this critical time is closer to dawn than to midnight.

Richard E. KRonauer

Division of Applied Sciences,

Harvard University,

Cambridge,

Massachusetts 02138, USA

Megan E. Jewett

Charles A. Czeisler

Laboratory for Circadian and Sleep

Disorders,

Department of Medicine,

Harvard Medical School,

Boston,

Massachusetts 02115, USA

1. Winfree, A. T. Nature 350, 18 (1991).

2. Jewett, M. E., Kronauer, R. E. \& Czeisler, C. A. Nature 350 59-62 (1991).

3. Winfree, A. T. J. theor. Biol. 28, 327--374 (1970).

4. Winfree, A. T. The Geometry of Biological Time 54-57 (Springer-Verlag, New York, 1980).

5. Gander, P. H. \& Lewis, R. D. Am. J. Physiol. 245, R10-17 (1983).

6. Daan S. \& Lewy, A. J. Psychopharmac. Bull. 20, 566-568 (1984).

\section{The right way}

SIR - In our recent paper on information storage using films of an azobenzene derivative $^{1}$, we proposed a mechanism to explain the experimental results which differed from that published by us in an earlier, preliminary paper ${ }^{2}$. After detailed investigations, we have concluded that the earlier mechanism was not adequate. It is the new mechanism proposed in ref. 1 , and described in more detail in ref. 3, that provides the possibility of photo-electrochemical information storage.

Z. F. Liv

K. HASHIMOTO

A. FUJISHIMA

Department of Synthetic Chemistry,

Faculty of Engineering,

University of Tokyo,

7-3-1 Hongo, Bunkyo-ku,

Tokyo 113, Japan

\footnotetext{
1. Liu, Z. F., Hashimoto, K. \& Fujishima, A. Nature 347 658-660 (1990)

2. Liu, Z. F., Hashimoto, K. \& Fujishima, A. J, electroanalyt Chem. 270, 437-443 (1989).

. 3. Liu, Z. F., Hashimoto, K. \& Fujishima, A. J. electroanalyt Chem. 297, 133-144 (1991)
} 